\title{
P04-6-28 Poster session
}

\section{Umbelliprenin efficiently induces complete pathologic response in 4T1 Tumor- bearing Balb/c Mice}

\author{
Mohsen Rashidi ${ }^{1}$, Ali Bazi ${ }^{2}$, Davar Amani ${ }^{3}$, Hamidreza Jamshidi ${ }^{3}$, Seyed Ali Ziai ${ }^{3}$ \\ ${ }^{I}$ Mazandaran University of Medical Scinces, Iran, ${ }^{2}$ Clinical Research Development Unit, Zabol University of Medical \\ Sciences, Zabol, Iran, ${ }^{3}$ Department of Pharmacology, School of Medicine, Shaheed Beheshti University of Medical \\ Sciences, Tehran, Iran
}

Background: Umbelliprenin (UMB) is a pharmacological agent with wide range activities in vitro. We investigated if UMB could induce pathological responses and affect tumor progression markers in 4T1 mammary-tumor-bearing mice.

Methods: Tumor-bearing female Balb/c mice were intraperitoneally administrated with 200 \&\#181;1 of UMB (12.5 $\mathrm{mg} / \mathrm{ml}$ ) for a period of 18 days. Tumor tissues as well as livers, kidney, and lungs were collected from all animals, fixed in formalin and embedded in paraffin, and then were subjected to the routine H\&E staining. Pathologic complete response (pCR) scoring system (based on remaining tumor cells, necrosis, mitosis, and pleomorphism rate) was adapted for pathologic examinations. Formalin-fixed and paraffin-embedded tumor-tissue slides were further subjected to immunohistochemical detection of Ki-67, CD31, CD45, VEGF, MMP2, MMP9, and E-Cadherin.

Results: According to the $\mathrm{pCR}$ scoring $(\mathrm{R}=0$; no reduction in malignant cells; $\mathrm{R}=1 ; 30 \%$ reduction in malignant cell, $\mathrm{R}=2$; $70 \%$ reduction in malignant cell, and $\mathrm{R}=3$, no malignant cells), the Tumor $+\mathrm{UMB}$ group revealed a significantly higher pCR score compared to Tumor + solvent and Tumor + NS groups $(\mathrm{P}<0.001)$. In immunohistochemical analysis of the tumors tissues, protein expression of Ki-67, CD31, VEGF, MMP2, and MMP9 was markedly decreased and that of ECadherin was markedly increased in tumor cells of UMB-treated group compared to the controls. No differences were observed between groups regarding the expression of CD45.

Conclusion: UMB treatment rendered significantly high tumor eradicating characteristics as revealed by pCR score. Accordingly, UMB significantly depressed expression of mitosis, and angiogenesis markers in tumor-bearing mice in vivo. 